\title{
Long-term prognostic impact of CT-Leaman score in patients with non- obstructive CAD: Results from the COronary CT Angiography EvaluatioN For Clinical Outcomes InteRnational Multicenter (CONFIRM) study
}

\author{
Daniele Andreini ${ }^{\mathrm{i}, *}$, Gianluca Pontone ${ }^{\mathrm{i}}$, Saima Mushtaq ${ }^{\mathrm{i}}$, Heidi Gransar ${ }^{\mathrm{d}}$, Edoardo Conte ${ }^{\mathrm{i}}$,
} Antonio L. Bartorelli ${ }^{i}$, Mauro Pepi ${ }^{i}$, Maksymilian P. Opolski ${ }^{a}$, Bríain ó Hartaigh ${ }^{\text {b }}$, Daniel S. Berman ${ }^{\text {d }}$, Matthew J. Budoff ${ }^{\mathrm{e}}$, Stephan Achenbach ${ }^{\mathrm{f}}$, Mouaz Al-Mallah ${ }^{\mathrm{g}}$, Filippo Cademartiri ${ }^{\mathrm{j}, \mathrm{y}}$, Tracy Q. Callister ${ }^{\mathrm{k}}$, Hyuk-Jae Chang ${ }^{1}$, Kavitha Chinnaiyan ${ }^{\mathrm{m}}$, Benjamin J.W. Chow ${ }^{\mathrm{n}}$, Ricardo Cury ${ }^{\circ}$, Augustin Delago ${ }^{\mathrm{q}}$, Martin Hadamitzky ${ }^{\mathrm{r}}$, Joerg Hausleiter ${ }^{\mathrm{s}}$, Gudrun Feuchtner ${ }^{\mathrm{t}}$, Yong-Jin Kim ${ }^{\mathrm{u}}$, Philipp A. Kaufmann ${ }^{\mathrm{v}}$, Jonathon Leipsic ${ }^{\mathrm{w}}$, Fay Y. Lin ${ }^{\mathrm{b}}$, Erica Maffei ${ }^{\mathrm{y}}$, Gilbert Raff ${ }^{\mathrm{m}}$, Leslee J. Shaw ${ }^{\mathrm{z}}$, Todd C. Villines ${ }^{\text {aa }}$, Allison Dunning ${ }^{\mathrm{c}}$, Hugo Marques ${ }^{\mathrm{h}}$, Ronen Rubinshtein ${ }^{\mathrm{p}}$, Niree Hindoyan ${ }^{\mathrm{x}}$, Millie Gomez ${ }^{\mathrm{x}}$, James K Min ${ }^{\mathrm{b}}$

${ }^{a}$ Department of Interventional Cardiology and Angiology, Institute of Cardiology, Warsaw, Poland

${ }^{\mathrm{b}}$ Department of Radiology, The New York-Presbyterian Hospital and the Weill Cornell Medical College, New York, NY, USA

${ }^{c}$ Department of Healthcare Policy and Research, Weill Cornell Medical College, New York, NY, USA

d Department of Imaging and Division of Cardiology, Department of Medicine, Cedars-Sinai Heart Institute, Cedars-Sinai Medical Center, Los Angeles, CA, USA

e Department of Medicine, Harbor UCLA Medical Center, Los Angeles, CA, USA

${ }^{\mathrm{f}}$ Department of Medicine, University of Erlangen, Erlangen, Germany

${ }^{g}$ King Saud bin Abdulaziz University for Health Sciences, King Abdullah International Medical Research Center, King Abdul Aziz Cardiac Center, Ministry of National Guard, Health Affairs, Saudi Arabia

${ }^{\text {h }}$ Department of Surgery, Curry Cabral Hospital, Lisbon, Portugal

i Department of Clinical Sciences and Community Health, University of Milan, Centro Cardiologico Monzino, IRCCS Milan, Italy

j Department of Radiology, Erasmus Medical Center, Rotterdam, The Netherlands

${ }^{\mathrm{k}}$ Tennessee Heart and Vascular Institute, Hendersonville, TN, USA

${ }^{1}$ Division of Cardiology, Severance Cardiovascular Hospital, Seoul, South Korea

${ }^{\mathrm{m}}$ William Beaumont Hospital, Royal Oaks, MI, USA

${ }^{\mathrm{n}}$ Department of Medicine and Radiology, University of Ottawa Heart Institute, Ontario, Canada

${ }^{\circ}$ Baptist Cardiac and Vascular Institute, Miami, FL, USA

${ }^{\mathrm{p}}$ Department of Cardiology at the Lady Davis Carmel Medical Center, The Ruth and Bruce Rappaport School of Medicine, Technion-Israel Institute of Technology, Haifa, Israel

${ }^{q}$ Capitol Cardiology Associates, Albany, NY, USA

${ }^{\mathrm{r}}$ Division of Cardiology, Deutsches Herzzentrum München, Munich, Germany

${ }^{\mathrm{s}}$ Medizinische Klinik I der Ludwig-Maximilians-Universität München, Munich, Germany

${ }^{t}$ Department of Radiology, Medical University of Innsbruck, Innsbruck, Austria

" Department of Medicine and Radiology, Seoul National University Hospital, Seoul, South Korea

v Department of Nuclear Cardiology, Cardiovascular Center, University Hospital Zurich, Zurich, Switzerland

w Department of Medical Imaging and Division of Cardiology, St. Paul's Hospital, University of British Columbia, Vancouver, BC, Canada

x Dalio Institute of Cardiovascular Imaging, Weill Cornell Medical College and New York-Presbyterian Hospital, New York, NY, USA

y Cardiovascular Imaging Unit, Giovanni XXIII Hospital, Monastier, Italy

${ }^{z}$ Department of Medicine, Emory University School of Medicine, Atlanta, GA, USA

aa Department of Medicine, Walter Reed National Medical Center, Bethesda, MD, USA

\section{A R T I C L E I N F O}

\section{Article history:}

Received 7 September 2016

Received in revised form 13 December 2016

Accepted 19 December 2016

Available online 28 December 2016

\begin{abstract}
A B S T R A C T
Background: Non-obstructive coronary artery disease (CAD) identified by coronary computed tomography angiography (CCTA) demonstrated prognostic value. CT-adapted Leaman score (CT-LeSc) showed to improve the prognostic stratification. Aim of the study was to evaluate the capability of CT-LeSc to assess long-term prognosis of patients with non-obstructive (CAD).

Methods: From 17 centers, we enrolled 2402 patients without prior CAD history who underwent CCTA that showed non-obstructive CAD and provided complete information on plaque composition. Patients were divided
\end{abstract}

\footnotetext{
it Relationships with industry. Dr. Min has served on the medical advisory boards Arineta; He is a consultant to Heart Flow and Cardiovascular Research Foundation; and has received research support from GE Healthcare.

* Corresponding author at: Via C. Parea 4, 20138 Milan, Italy.

E-mail address: daniele.andreini@ccfm.it (D. Andreini).
} 
Keywords:

Coronary CT angiography

CT-adapted Leaman score

Non-obstructive CAD

Prognosis

Patients reclassification into a group without CAD and a group with non-obstructive CAD ( $<50 \%$ stenosis). Segment-involvement score (SIS) and CT-LeSc were calculated. Outcomes were non-fatal myocardial infarction (MI) and the combined end-point of MI and all-cause mortality.

Results: Patient mean age was $56 \pm 12$ years. At follow-up (mean $59.8 \pm 13.9$ months), 183 events occurred (53 MI, 99 all-cause deaths and 31 late revascularizations). CT-LeSc was the only multivariate predictor of MI (HRs 2.84 and 2.98 in two models with Framingham and risk factors, respectively) and of MI plus all-cause mortality (HR 2.48 and 1.94 in two models with Framingham and risk factors, respectively). This was confirmed by a net reclassification analysis confirming that the CT-LeSc was able to correctly reclassify a significant proportion of patients (cNRI 0.28 and 0.23 for MI and MI plus all-cause mortality, respectively) vs. baseline model, whereas SIS did not. Conclusion: CT-LeSc is an independent predictor of major acute cardiac events, improving prognostic stratification of patients with non-obstructive CAD.

(c) 2016 Elsevier Ireland Ltd. All rights reserved.

\section{Introduction}

In recent years, studies supporting the prognostic value of coronary CT angiography (CCTA), including single-center studies and a large multicenter registry, have been published [1,2]. According to these data, while the absence of identifiable plaques in the coronary tree is associated with an excellent prognosis, it has also been consistently demonstrated that the identification of non-obstructive lesions, a unique feature of CCTA as a non-invasive coronary imaging modality, has prognostic value. This has clinical implications because many patients fall in this category, as reflected by the high proportion of patients with atherosclerotic plaques in many CCTA databases [2-5]. Nevertheless, as non-obstructive CAD is a very heterogeneous and prevalent condition, there is the need for tools to quantify total coronary atherosclerotic burden in order to better stratify these patients. Recently, a new developed score, the CTadapted Leaman score (CT-LeSc), using the comprehensive information on lesion localization, plaque composition and degree of stenosis provided by CCTA, resulted in a relatively small, single-center setting, to be an independent long-term predictor of hard cardiac events and to improve the CCTA prognostic stratification of non-obstructive CAD [6].

In the present prospective international multicenter study, we evaluated the capability of the CT-LeSc to stratify the long-term prognosis of a large cohort of patients with non-obstructive CAD at CCTA evaluation.

\section{Methods}

\subsection{Study population}

The design and rationale of the CONFIRM (COronary CT Angiography EvaluatioN For Clinical Outcomes: An InteRnational Multicenter) registry has been described previously [7]. For the current study, we utilized the data from the CONFIRM long-term follow-up registry that included only patients who had a follow-up duration of more than three years. Overall, 17,181 patients who underwent CCTA at 17 centers in 9 countries (Austria, Canada, Germany, Israel, Italy, Portugal, South Korea, Switzerland, and United States) were enrolled between February 2003 and May 2011 for long-term follow-up. Inclusion criteria were age $>18$ years, a CCTA performed with a scanner equipped with at least 64-detectors, and CCTA images of interpretable quality. Among the 5010 patients in whom MACE data at follow-up and complete plaque characteristics data were collected we excluded those with prior history of CAD ( $n=1741)$, myocardial revascularization performed early after CCTA ( $<90$ days) $(n=377)$ and presence of obstructive coronary lesions $(>50 \%)(n=490)$. The analytic sample comprised 2402 . patients. Informed consent was obtained from each patient and [2] the study protocol conforms to the ethical guidelines of the 1975 Declaration of Helsinki as reflected in a priori approval by the institution's human research committee.

\subsection{Risk factor assessment}

Clinical CAD risk factors including smoking, hypertension, dyslipidemia, diabetes, and family history were collected prior to CCTA examination by direct patient interview performed by a physician or nurse research coordinator and/or with standardized site questionnaires [7].

\subsection{Imaging analysis}

CCTA data were acquired using multi-detector row CT scanners consisting of 64-rows or greater. Expert readers analyzed all CCTA images according to the guidelines of the Society of Cardiovascular Computed Tomography (SCCT) $[8,9]$. We defined coronary atherosclerosis in CCTA images as any tissue structure larger than $1 \mathrm{~mm}^{2}$, which was either within the lumen of the coronary artery or adjacent to the coronary artery lumen and could be distinguished from the adjacent epicardial fat, pericardial tissue, or the artery lumen. We used a modified American Heart Association 16-segment coronary artery tree model for analysis [10]. Coronary artery luminal narrowing was defined as the presence of any plaque resulting in a $\%$ diameter reduction $>0$. Non-obstructive lesions were defined as coronary artery segments showing plaques with a luminal diameter stenosis $<50 \%$. Normal CCTA was defined as the absence of any coronary artery luminal narrowing. The SIS, ranging from 0 to 16 , was calculated as the total number of segments with plaques (any degree of stenosis). The methodology for the CT-LeSc has been previously described [11]. Briefly, three sets of weighting factors are used for this score: 1 ) localization of the coronary plaques, accounting for dominance; 2) type of plaque, with a multiplication factor of 1 for calcified plaques and of 1.5 for non-calcified and mixed plaques; and 3) degree of stenosis, with a multiplication factor of 0.615 for non-obstructive $(<50 \%$ stenosis $)$ and a multiplication factor of 1 for obstructive ( $\geq 50 \%$ stenosis) lesions. The CT-LeSc was calculated on a patient level as the sum of the partial CT-LeSc of all evaluable coronary segments. For both the SIS and the CT-LeSc, prognostically validated cut-off values $(>5)$ were used $[4,6]$. Analysis of coronary artery calcium score was performed when available. The total mean dose length product for CCTA was estimated to be $938 \pm 379 \mathrm{mGy} \times \mathrm{cm}$, corresponding to an estimated radiation dose of $13 \pm 5 \mathrm{mSv}$.

\subsection{Patient follow-up}

The primary outcomes of the current study were non-fatal myocardial infarction (MI) and the combined end-point of MI and all-cause mortality. As previously reported [7], the outcomes were assessed at each institution by direct interview, telephone contact, review of medical records, or using a mailed standardized questionnaire. In the USA, all-cause mortality was additionally searched by the Social Security Death Index. Site physicians defined MI according to ACC/AHA guidelines and the World Health Organization Universal Definition of Myocardial Infarction [12]. All revascularizations were recorded and patients with elective myocardial revascularization were censored at follow-up. 


\subsection{Statistical analysis}

Categorical variables are presented as counts and proportions. Continuous variables are presented as means \pm SD. A one-way ANOVA or the Kruskal-Wallis test was used to conduct continuous variables intergroup comparisons among patients without CAD, nonobstructive CAD but a LS $<5$ and non-obstructive CAD but a $\mathrm{LS}>5$. Pearson's chi-square test $\left(\chi^{2}\right)$ was used for categorical variables comparison. Time-to-event analysis for the study endpoints were calculated using univariable Cox proportional-hazards models reporting hazard ratios (HR) with 95\% confidence intervals (95\% CI). Multivariable Cox proportional hazards models were also constructed with variables based on clinical judgment univariate analysis results. All the analyses were performed evaluating combined endpoints (MI, MI plus all cause of death,). To avoid overfitting and multicollinearity issues, we developed four different models, for all different combined endpoints. The first model was adjusted for the CT-LeSc and the Framingham risk score. The second model was adjusted for the SIS and the Framingham risk score. The third model was adjusted for the CT-LeSc and baseline clinical characteristics. The fourth model was adjusted for the SIS and baseline clinical characteristics.

A sub-analysis were performed in patients in which CACS was available; a prognostically validated cut-off $>400$ was used as previously suggested [13]. Moreover we performed a separate sub-analysis for patients with and without chest pain at baseline. Of note, in the analysis of symptomatic subjects the Morise score was included in model 1 and 2, instead of the Framingham score.

Survival curves were calculated using the Kaplan-Meier method for population stratified by the presence of non-obstructive CAD and the CT-LeSc, with each survival curve compared using the log-rank test. A two-tailed $p$ value of $<0.05$ was considered statistically significant. The comparison between performance of the CT-LeSc and SIS added to a baseline model was further quantified by a continuous net reclassification index (cNRI) [14].

\section{Results}

\subsection{Patient characteristics and MACE}

Indications for CCTA were chest pain (1200 patients, 49.9\%), multiple CAD risk factors ( 595 patients, $24.7 \%$ ), and equivocal or abnormal stress test results (607 patients, $25.3 \%$ ). Mean pre-test probability of CAD was low-to-intermediate (mean Morise score $11.6 \pm 4.2$ ). The mean duration of follow-up was $59.8 \pm 13.9$ months, up to 96 months (Table 1 ). One-hundred and eighty-three patients exhibited events during follow-up (53 MI, 99 all-cause deaths and 31 late revascularizations).

\subsection{Univariate predictors of events}

Among clinical characteristics, therapy with aspirin was the only predictor of MI, whereas hypertension and diabetes were predictors of MI plus all-cause death. Among CCTA data, a SIS $>5$ and non-obstructive CAD with a CT-LeSc $>5$ were predictors of MI, whereas a SIS $>5$, nonobstructive CAD with a CT-LeSc $\leq 5$ and non-obstructive CAD with a CT-LeSc $>5$ were predictors of MI plus all-cause death (Table 2).

\subsection{Multivariate predictors of events}

The only significant independent predictor of MI was nonobstructive CAD with a CT-LeSc > 5 (HR 2.84 and 2.98 in model 1 and model 3, respectively). The independent predictors of MI plus allcause death were the Framingham score (HR 1.02 and 1.03 in model 1 and model 2, respectively), age (HR 1.04 and 1.05 in model 3 and model 4, respectively), diabetes (HR 1.84 and 1.86 in model 3 and model 4, respectively), a SIS $>5$ (HR 1.95 in model 2, HR not significant in model 4), non-obstructive CAD with a CT-LeSc $\leq 5$ (HR 2.05 and 1.55 in model 1 and model 3, respectively) and non-obstructive CAD with a CT-LeSc $>5$ (HR 2.48 and 1.94 in model 1 and model 3, respectively) (Table 3).

Table 1

Clinical and CCTA baseline characteristics.

\begin{tabular}{|c|c|c|c|c|}
\hline & $\begin{array}{l}\text { All patients } \\
(n=2402)\end{array}$ & $\begin{array}{l}\text { No CAD } \\
(n=1450)\end{array}$ & $\begin{array}{l}\text { Non-obstructive CAD with } \\
\text { LS } \leq 5(n=611)\end{array}$ & $\begin{array}{l}\text { Non-obstructive CAD with } \\
\text { LS }>5(n=341)\end{array}$ \\
\hline \multicolumn{5}{|l|}{ Clinical characteristics } \\
\hline Age & $56 \pm 12$ & $53 \pm 12$ & $61 \pm 10^{*}$ & $63 \pm 11^{*}, \varsigma$ \\
\hline Male & $1208(50.3)$ & $657(45.3)$ & $346(56.7)^{*}$ & $205(60.1)^{*}$ \\
\hline BMI & $28 \pm 5.49$ & $27.79 \pm 5.39$ & $28.09 \pm 5.11$ & $28.61 \pm 6.40$ \\
\hline Hypertension & $1302(54.2)$ & $674(46.5)$ & $394(64.5)^{*}$ & $234(68.6)^{*}$ \\
\hline Diabetes & $252(10.5)$ & $116(8)$ & $74(12.1)^{\S}$ & $62(18.1)^{*}, \mathcal{C}$ \\
\hline Current smoking & $425(17.7)$ & $250(17.2)$ & $105(17,2)$ & $70(20.53)$ \\
\hline Family history & $798(33.2)$ & $492(33.9)$ & $188(30,8)$ & $118(34.6)$ \\
\hline Dyslipidemia & $1207(50.3)$ & $641(44.2)$ & $342(56)^{*}$ & $224(65.7)^{*}, \mathcal{c}$ \\
\hline Morise & $11.57 \pm 4.16$ & $10.70 \pm 4.42$ & $12.68 \pm 3.37^{*}$ & $13.19 \pm 3.31^{*}$ \\
\hline Framingham & $11.99 \pm 9.63$ & $9.74 \pm 7.78$ & $14.83 \pm 10.46^{*}$ & $16.27 \pm 11.98^{*}$ \\
\hline \multicolumn{5}{|l|}{ Chest pain at baseline } \\
\hline No chest pain & $1053(43.8)$ & $590(40.7)$ & $308(50.4)$ & $155(45.4)$ \\
\hline Non-cardiac/unspecified pain & $418(17.4)$ & $296(20.4)$ & $79(12.9)$ & $43(12.6)$ \\
\hline Atypical chest pain & $737(30.7)$ & $436(30.1)$ & $184(30.1)$ & $117(34.3)$ \\
\hline Typical chest pain & $194(8.1)$ & $128(8.8)$ & $40(6.5)$ & $26(7.6)$ \\
\hline \multicolumn{5}{|l|}{ Therapy } \\
\hline ASA & $503(20.9)$ & $265(18.3)$ & $137(22.4)^{\S}$ & $101(29.6)^{*}$, ç \\
\hline Statin & $506(21.1)$ & $226(15.6)$ & $161(26.4)^{*}$ & $119(34.8)^{*}, \mathrm{c}$ \\
\hline \multicolumn{5}{|l|}{ CCTA characteristics } \\
\hline SIS & $1.08 \pm 1.89$ & 0 & $1.61 \pm 0.88^{*}$ & $4.64 \pm 2.36^{*}, 0$ \\
\hline
\end{tabular}

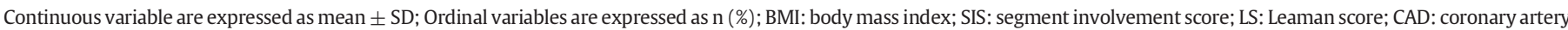
disease.

\footnotetext{
* $p<0,0001$ vs no CAD.

o $p<0,0001$ vs $\mathrm{LS}<5$.

$\S p<0,05$ vs no CAD.

c $p<0,05$ vs $\mathrm{LS}<5$.
} 
Table 2

Univariate analysis.

\begin{tabular}{|c|c|c|c|c|}
\hline & \multirow{2}{*}{$\begin{array}{l}\text { MI } \\
\mathrm{HR}(95 \% \mathrm{CI})\end{array}$} & \multicolumn{3}{|c|}{$\mathrm{MI}+$ all-cause death } \\
\hline & & $p$ & $\mathrm{HR}(95 \% \mathrm{CI})$ & $p$ \\
\hline \multicolumn{5}{|l|}{ Clinical characteristics } \\
\hline Age & $1(0.98-1.02)$ & 0.871 & $1.04(1.02-1.05)$ & $<0.0001$ \\
\hline Male & $0.78(0.46-1.32)$ & 0.343 & $1.1(0.79-1.51)$ & 0.5731 \\
\hline BMI & $1.03(0.99-1.07)$ & 0.128 & $1.01(0.98-1.04)$ & 0.596 \\
\hline Hypertension & $1.52(0.87-2.67)$ & 0.143 & $1.97(1.39-2.79)$ & $<0.0001$ \\
\hline Diabetes & $1.85(0.9-3.79)$ & 0.095 & $1.95(1.29-2.96)$ & 0.002 \\
\hline Current smoking & $1.68(0.91-3.1)$ & 0.096 & $1.33(0.91-1.95)$ & 0.144 \\
\hline Family history & $1.46(0.84-2.52)$ & 0.182 & $0.86(0.1-1.23)$ & 0.417 \\
\hline Dyslipidemia & $1.17(0.68-2.01)$ & 0.573 & $0.9(0.65-1.24)$ & 0.505 \\
\hline Morise & $0.98(0.92-1.05)$ & 0.629 & $1.03(0.99-1.07)$ & 0.168 \\
\hline Framingham & $1.01(0.99-104)$ & 0.366 & $1.03(1.02-1.04)$ & $<0.0001$ \\
\hline \multicolumn{5}{|l|}{ Chest pain at baseline } \\
\hline No chest pain & 1 & & 1 & \\
\hline Non-cardiac pain/unspecified & $1.34(0.69-2.64)$ & 0.388 & $0.80(0.52-1.24)$ & 0.325 \\
\hline Atypical chest pain & $0.82(0.42-1.57)$ & 0.547 & $0.50(0.33-0.75)$ & 0.001 \\
\hline Typical chest pain & $1.07(0.41-2.80)$ & 0.886 & $0.60(0.31-1.16)$ & 0.130 \\
\hline \multicolumn{5}{|l|}{ Therapy } \\
\hline ASA & $1.84(1.01-3.34)$ & 0.046 & $1.42(0.98-2.05)$ & 0.063 \\
\hline Statin & $1.2(0.63-2.27)$ & 0.585 & $1.12(0.77-1.63)$ & 0.563 \\
\hline \multicolumn{5}{|l|}{ CCTA characteristics } \\
\hline SIS $>5$ & $1.13(1.02-1.26)$ & 0.022 & $1.18(1.12-1.25)$ & $<0.001$ \\
\hline No CAD & 1 & & 1 & \\
\hline Non-Ob CT-LeSc $\leq 5$ & $1.18(0.62-2.23)$ & 0.618 & $1.88(1.3-2.72)$ & 0.0008 \\
\hline Non-OB CT-LeSc $>5$ & $2.11(1.11-3.99)$ & 0.023 & $2.37(1.58-3.57)$ & $<0.001$ \\
\hline
\end{tabular}

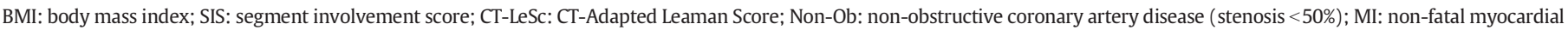
infarction; CAD: coronary artery disease.

Table 3

Multivariate analysis.

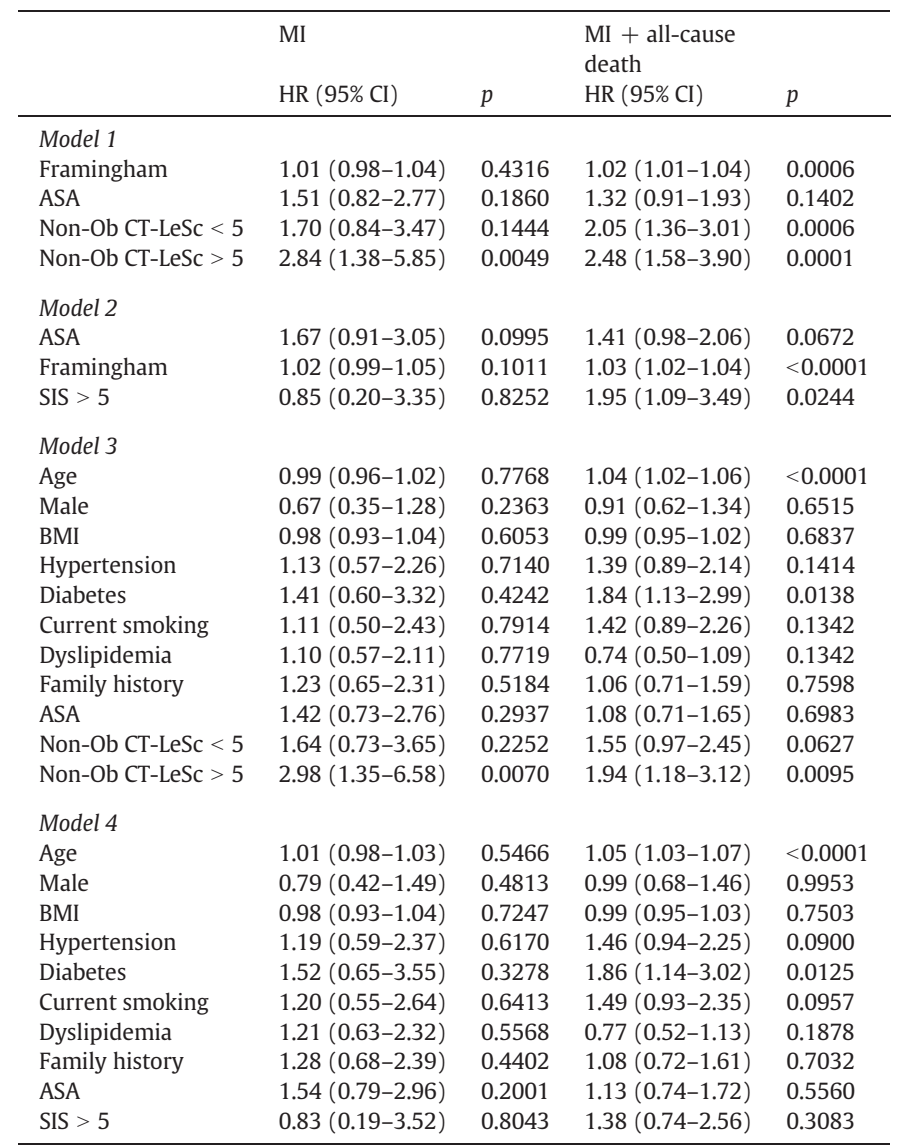

\subsection{Survival analysis}

When MI only was considered as outcome, the event-free survival rates were $98 \%$ in patients without CAD, $98 \%$ in patients with nonobstructive CAD and a CT-LeSc $\leq 5$ and 95\% in patients with nonobstructive CAD and a CT-LeSc $>5$ (log-rank $p$ value $=0.01$ ) (Figure 1A). When the end-point of MI plus all-cause mortality was used, event-free survival rates were $97 \%, 92 \%$ and $88 \%$ in patients without CAD, with non-obstructive CAD and a CT-LeSc $\leq 5$ and with non-obstructive CAD and a CT-LeSc $>5$, respectively (log-rank $p$ value $<0.0001$ ) (Figure 1B).

\subsection{Reclassification index}

The net reclassification analysis (Table 4 ) showed that a CT-LeSc $>5$ is able to correctly reclassify a significant proportion of patients $(0.28$ and 0.23 for MI and MI plus all-cause mortality, respectively), in comparison with the baseline model including age, male gender, diabetes, hypertension, smoking, dyslipidemia, and family history of premature CAD. Conversely, reclassification using a SIS $>5$ was not statistically significant in any models.

\subsection{Coronary artery calcium score sub-analysis}

Coronary artery calcium score (CACS) was available in 1537 patients (64\%). CACS $>400$ was identified in 67 patients $(4.4 \%)$ in the entire cohort; CACS $>400$ was present in 6 patients $(1.6 \%)$ with CT LeSc $\leq 5$ and in 61 subjects (22.9\%) among those with CT LeSc $>5$. At univariate analysis neither CACS > 400 or SIS $>5$ was associated with MI (HR 1.85; $95 \% \mathrm{CI} 0.57-5.98, p=0.306$ and $0.89 ; 0.22-3.68, p=0.896$ ), while only CT-LeSc $>5$ was a significantly predictors of MI (HR 2.09; $95 \%$ CI $1.02-$ $4.30, p=0.04)$. When the composite endpoint including MI and all cause of death was considered, CACS $>400$, SIS $>5$ and CT-LeSc $>5$ 

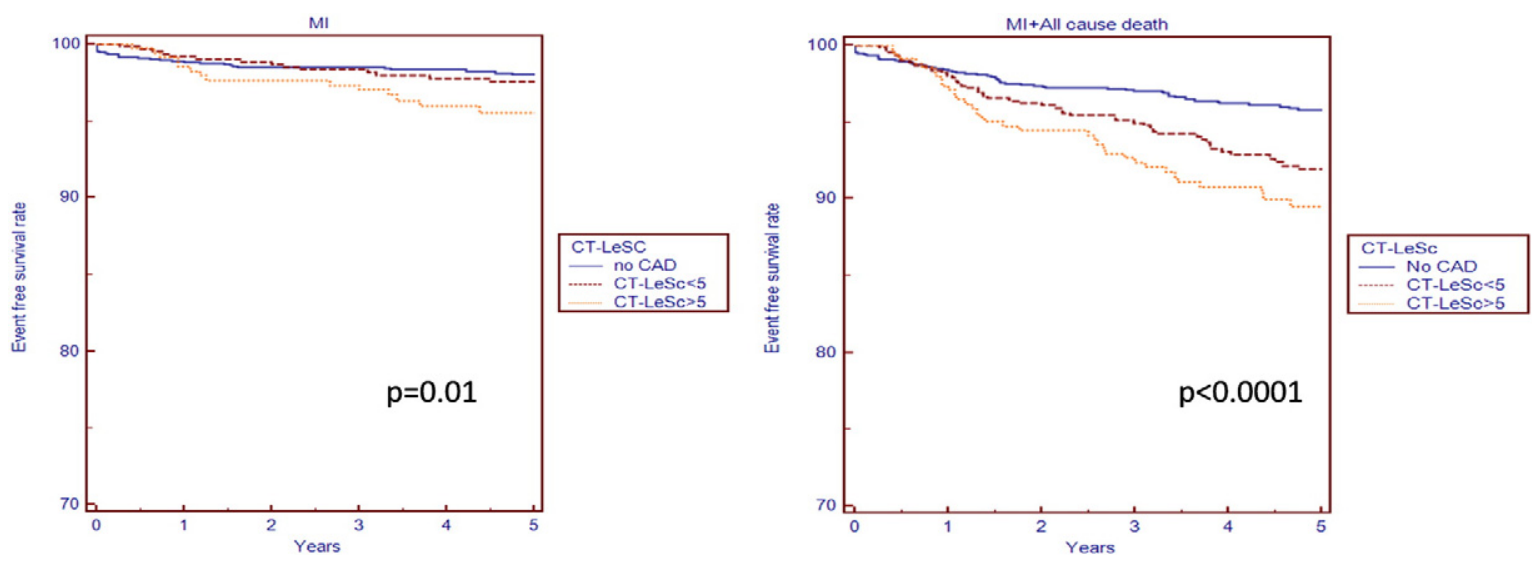

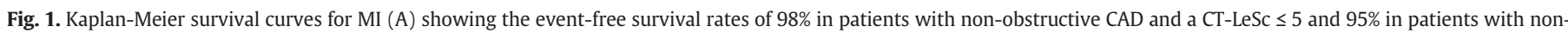

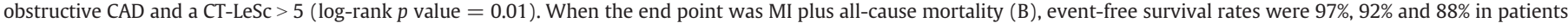

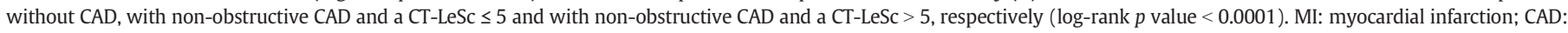
coronary artery disease; CT-LeSc: computed tomography adapted Leaman score.

were all significative predictors of events (HR 3.99, HR 2.38 and HR 2.73, respectively; $p<0.001$ ).

At multivariate analysis CACS $>400$ remains a significantly predictor of MI + all cause of death when adjusted for Framingham risk score (HR $3.04 ; 95 \% \mathrm{CI} 1.72-5.36, p<0.001)$ and for baseline clinical characteristics (HR 2.05; 95\% CI 1.14-3.68, $p=0.017$ ); SIS > 5 remains significantly associated to MI + all cause of death only when adjusted for Framingham risk score (HR 1.96; 95\% CI 1.09-3.55, $p=0.027$ ), but not when adjusted for clinical baseline characteristics.

Of note, only CT-LeSc $>5$ was significantly associated to MI when adjusted both for Framingham risk score (HR 2.68; 95\% CI 1.18-6.12, $p=$ 0.019 ) and for baseline clinical characteristics (HR 3.13; 95\% CI 1.29$7.53, p=0.012$ ) at multivariate analysis. When the composite endpoint including MI + all cause of death was considered CL-LeSc $>5$ was still associated to events both when adjusted for Framingham risk score (HR 2.52; 95\% CI 1.52-4.16, $p<0.001$ ) and for baseline characteristics (HR 1.79, 95\% CI 1.05-3.05, $p=0.038$ ).

In this subgroup of patients with CACS reported, the event free survival rates were $98 \%$ in those with CT-LeSc $<5$ and $95 \%$ in those with CTLeSc $>5(\log$-rank $p=0.0289)$ (Figure $2 \mathrm{~B})$; on the contrary CACS $>400$ score was not significantly associated to worst survival rates (Figure 2A).

\subsection{Asymptomatic patients vs symptomatic patients}

Among the entire cohort 1053 patients (43.8\%) did not report chest pain at baseline, while 1349 patients (56.2\%) were symptomatic at the time of CCTA.

A separate sub-analysis in asymptomatic subjects showed that at univariate analysis there were no predictor of MI; on the contrary, age, hypertension, diabetes and the Framingham score were the clinical characteristics associated to MI + all cause of death, while among CT parameters both SIS $>5$ and CT-LeSc $>5$ were associated to MI + all cause of death (Table 5 ).

Table 4

Net Reclassification Index for SIS $>5$ and CT-LeSc $>5$ for prediction of composite endpoints.

\begin{tabular}{llllllll}
\hline & & \multicolumn{2}{l}{ MI } & & \multicolumn{3}{l}{ MI + all-cause death } \\
\cline { 5 - 7 } & cNRI & cNRI 95\% CI & $p$ & & cNRI & cNRI 95\% CI & $p$ \\
\hline $\mathrm{BL}$ & - & - & - & - & - & - \\
$\mathrm{BL}+\mathrm{SIS}>5$ & 0.15 & $-0.09-0.40$ & 0.271 & -0.039 & $-0.19-0.11$ & 0.648 \\
$\mathrm{BL}+$ CT-LeSc $>5$ & 0.28 & $0.02-0.54$ & 0.046 & 0.23 & $0.07-0.39$ & 0.006 \\
\hline
\end{tabular}

In the subgroups of patients with chest pain at baseline only CTLeSc $>5$ was associated to MI at univariate (HR 2.5; 95\% CI 1.13-5.75, $p=0.025$ ), while age, hypertension and the Morise score were associated to MI + all cause of death, but only CT-LeSc > 5 (HR 2.53; 95\% Cl $1.42-4.48, p=0.002$ ) was found to be associated to this composite end-point among CCTA variables (Table 6).

At multivariate analysis SIS $>5$ was not associated to endpoints both in asymptomatic and symptomatic patients. On the contrary CT-LeSc $>5$ was found to be a predictor of MI in symptomatic patients ( HR 2.63 and 2.76 in Model 1 and 3, respectively) and to be associated with MI + All cause of death both in asymptomatic and symptomatic patients only when adjusted for Framingham or Morise score (HR 2.56 and 2.39 in asymptomatic and symptomatic patients, respectively), but not when clinical baseline characteristics were evaluated separately (Table 7).

\section{Discussion}

Coronary CTA has been demonstrated to be accurate for the detection of non-obstructive CAD and coronary atherosclerosis when compared to coronary intravascular ultrasound [15]. Detection of nonobstructive $\mathrm{CAD}$, which may be considered an unique feature of CCTA among other non-invasive imaging modalities, has relevant prognostic implications. Indeed, it may identify a population that has an higher event-free survival rate as compared to that of patients with obstructive disease but lower than that of patients with normal coronaries $[4,5,16$, 17]. In order to better stratify the prognosis among the large and heterogeneous cohort of patients with non-obstructive CAD, different coronary plaque scores have been proposed. Among them, the SIS and SSS demonstrated a remarkable prognostic value $[4,18]$. Particularly, a single-center study by Bittencourt et al. demonstrated that among patients with obstructive CAD, a greater extent of non-obstructive plaques, as quantified by SIS, was associated with a higher event rate [16].

\subsection{CT adapted-Leaman score}

A recently proposed plaque score, the CT-LeSc based on lesion localization, plaque composition and degree of stenosis, demonstrated to improve the prognostic stratification of non-obstructive CAD in an another single-center study [6]. To the best of our knowledge, this is the first prospective international multicenter study finalized to evaluate if the CT-LeSc is able to stratify the long-term prognosis in a selected but large patient cohort with non-obstructive lesions. The main findings of the study are that the CT-LeSc allows to distinguish within patients with non-obstructive CAD those with a cardiac event risk similar to that shared by patients without plaques from those with a less favorable 

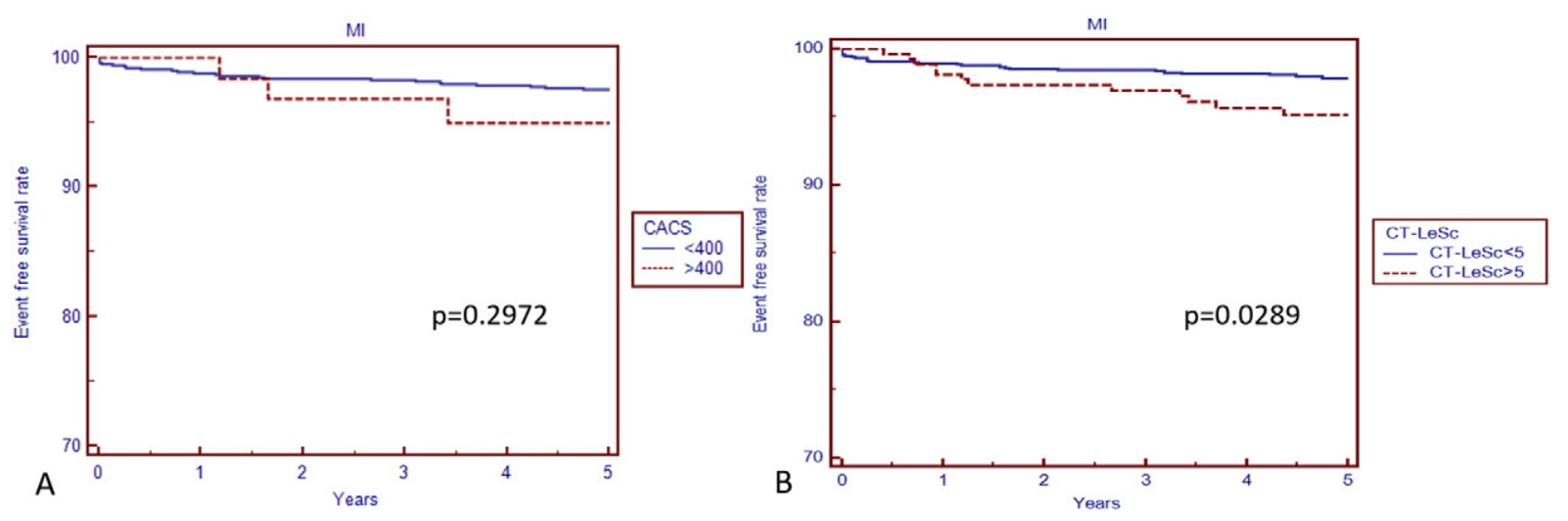

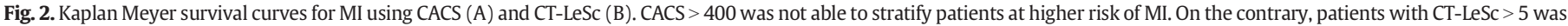

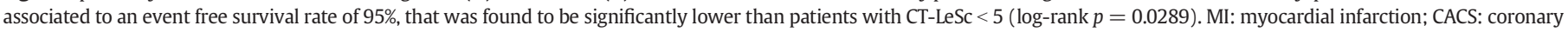
artery calcium score; CT-LeSc: computed tomography adapted Leaman score.

prognosis. Moreover, the CT-LeSc appears to be superior to the SIS, despite the latter already showed to possess a good prognostic value. In particular, analyzing the primary outcomes of the study we found that a CT-LeSc $>5$ was an univariate predictor of MI and MI plus all-cause death and the only multivariate predictor of MI.

\subsection{CT-LeSc vs SIS}

The CT-LeSc appears to be superior to the SIS that exhibited HR systematically lower than the CT-LeSc at both univariate and multivariate analysis. This is confirmed by the net reclassification analysis showing that the CT-LeSc, but not the SIS, was able to correctly reclassify a significant proportion of patients vs. a baseline model including age, gender and risk factors for both primary and secondary endpoints.

\subsection{CACS and CT-LeSC}

In the sub-group of patients with calcium score, CACS $>400$ has been confirmed to be a predictor of composite end-point including all cause of death, but only CT-LeSc appeared to correctly identify patients at higher risk of MI. These findings could be explained by recent studies, suggesting that non-calcified plaques may be associated to higher risk of acute coronary syndrome when compared to calcified ones [19].

Table 5

Univariate analysis for asymptomatic patients.

\begin{tabular}{|c|c|c|c|c|}
\hline & \multirow{2}{*}{$\begin{array}{l}\text { MI } \\
\text { HR (95\% CI) }\end{array}$} & \multicolumn{3}{|c|}{$\mathrm{MI}+$ all-cause death } \\
\hline & & $p$ & $\mathrm{HR}(95 \% \mathrm{CI})$ & $p$ \\
\hline \multicolumn{5}{|l|}{ Clinical characteristics } \\
\hline Age & $1.01(0.98-1.04)$ & 0.578 & 1.03 (1.01-1.05) & 0.001 \\
\hline Male & $0.68(0.31-1.52)$ & 0.356 & $0.93(0.60-1.43)$ & 0.717 \\
\hline BMI & $1.04(0.98-1.09)$ & 0.182 & $1.02(0.98-1.05)$ & 0.424 \\
\hline Hypertension & $1.63(0.70-3.78)$ & 0.261 & $1.83(1.15-2.94)$ & 0.011 \\
\hline Diabetes & $1.43(0.43-4.79)$ & 0.556 & $2.05(1.15-3.63)$ & 0.015 \\
\hline Current smoking & $0.99(0.34-2.89)$ & 0.996 & $0.98(0.55-1.75)$ & 0.962 \\
\hline Family history & $1.26(0.54-2.93)$ & 0.597 & $0.77(0.46-1.29)$ & 0.333 \\
\hline Dyslipidemia & $0.62(0.27-1.42)$ & 0.263 & $0.67(0.40-1.01)$ & 0.052 \\
\hline Framingham & $0.98(0.93-1.03)$ & 0.404 & $1.02(1.01-1.03)$ & 0.048 \\
\hline \multicolumn{5}{|l|}{ Therapy } \\
\hline ASA & $1.50(0.56-3.99)$ & 0.411 & $1.69(1.03-2.77)$ & 0.038 \\
\hline Statin & $0.70(0.20-2.40)$ & 0.572 & $0.97(0.55-1.72)$ & 0.931 \\
\hline \multicolumn{5}{|l|}{ CCTA characteristics } \\
\hline No CAD & 1 & & 1 & \\
\hline SIS $>5$ & $1.94(0.46-8.22)$ & 0.367 & $2.02(1.06-4.56)$ & 0.034 \\
\hline Non-Ob CT-LeSc $\leq 5$ & $1.12(0.44-2.85)$ & 0.807 & $2.11(1.29-3.42)$ & 0.003 \\
\hline Non-OB CT-LeSc $>5$ & $1.61(0.56-4.53)$ & 0.376 & $2.17(1.22-3.86)$ & 0.009 \\
\hline
\end{tabular}

\subsection{Asymptomatic and symptomatic patients}

Approximately half of the patients included in this study were asymptomatic for chest pain at the time of CCTA. In order to better clarify the possible role of CCTA in the evaluation of asymptomatic patients we performed a specific separate analysis in asymptomatic vs symptomatic patients. Of note we have found no clinical or CCTA parameters resulting to be significantly associated to $\mathrm{MI}$ in asymptomatic patients. On the contrary in symptomatic patients only CT-LeSc $>5$ was associated to MI both at univariate and multivariate analyses. This findings suggest that CT-LeSc could be an important tool to discriminate patients at higher risk of MI among those symptomatic for chest pain, even if CCTA results to be negative for significative stenosis ( $>50 \%)$.

\subsection{Survival analysis}

Analyzing the survival curves for primary endpoints, we found that MI-free survival rate was $98 \%$ for patients with normal coronary arteries and for those with non-obstructive CAD and a CT-LeSc $\leq 5$. Conversely, the survival rate fell to $95 \%$ in the presence of a CT-LeSc $>5$, confirming the power of the CT-LeSc in stratifying the prognosis of patients with non-obstructive lesions. Adding all-cause mortality to MI in the survival analysis, patients with non-obstructive stenosis and a low CT-LeSc

Table 6

Univariate analysis for symptomatic patients.

\begin{tabular}{lllll}
\hline & MI & \multicolumn{3}{l}{ MI + All-cause death } \\
\cline { 4 - 5 } & HR $(95 \% \mathrm{CI})$ & $p$ & HR $(95 \% \mathrm{CI})$ & $p$ \\
\hline Clinical characteristics & & & & \\
Age & $1.01(0.97-1.03)$ & 0.921 & $1.04(1.02-1.07)$ & $<0.001$ \\
Male & $0.86(0.43-1.74)$ & 0.862 & $0.74(0.46-1.22)$ & 0.246 \\
BMI & $1.02(0.95-1.08)$ & 0.641 & $1.01(0.96-1.05)$ & 0.754 \\
Hypertension & $1.15(0.57-2.31)$ & 0.696 & $1.76(1.06-2.95)$ & 0.031 \\
Diabetes & $1.93(0.9-4.67)$ & 0.149 & $1.83(0.98-3.42)$ & 0.059 \\
Current smoking & $1.49(0.67-3.32)$ & 0.323 & $1.39(0.79-2.44)$ & 0.249 \\
Family history & $1.38(0.68-2.79)$ & 0.373 & $1.05(0.63-1.72)$ & 0.866 \\
Dyslipidemia & $1.51(0.74-3.08)$ & 0.259 & $1.40(0.87-2.29)$ & 0.181 \\
Morise & $1.01(0.92-1.09)$ & 0.946 & $1.08(1.02-1.16)$ & 0.016 \\
Therapy & & & & \\
ASA & $1.82(0.84-3.93)$ & 0.129 & $1.18(0.66-2.08)$ & 0.571 \\
Statin & $1.31(0.59-2.87)$ & 0.513 & $1.29(0.76-2.18)$ & 0.354 \\
CCTA characteristics & & & & \\
No CAD & 1 & & 1 & \\
SIS & - & - & $2.03(1.01-5.31)$ & 0.051 \\
Non-Ob CT-LeSc $\leq 5$ & $1.20(0.49-2.92)$ & 0.677 & $1.43(0.80-2.57)$ & 0.229 \\
Non-OB CT-LeSc $>5$ & $2.54(1.13-5.75)$ & 0.025 & $2.53(1.42-4.48)$ & 0.002 \\
\hline
\end{tabular}


Table 7

Multivariate analysis for asymptomatic and asymptomatic patients.

\begin{tabular}{|c|c|c|c|c|}
\hline & $\begin{array}{l}\text { MI } \\
\text { HR (95\% CI) }\end{array}$ & $p$ & $\begin{array}{l}\text { MI + all-cause death } \\
\mathrm{HR}(95 \% \mathrm{CI})\end{array}$ & $p$ \\
\hline $\begin{array}{l}\text { Asymptomatic patients } \\
\text { Model } 1^{*}\end{array}$ & & & & \\
\hline $\begin{array}{l}\text { Non-Ob CT-LeSc }>5 \\
\text { Model 2* }\end{array}$ & $3.34(0.97-11.44)$ & 0.059 & $2.56(1.31-4.99)$ & 0.006 \\
\hline $\begin{array}{r}\text { SIS }>5 \\
\text { Model } 3^{\S}\end{array}$ & $2.42(0.55-10.73)$ & 0.248 & $1.80(0.82-3.95)$ & 0.144 \\
\hline $\begin{array}{l}\text { Non-Ob CT-LeSc > } 5 \\
\text { Model } 4^{\S}\end{array}$ & $3.15(0.84-11.78)$ & 0.089 & $1.50(0.73-2.94)$ & 0.227 \\
\hline SIS $>5$ & $1.19(0.26-5.51)$ & 0.821 & $0.98(0.41-2.35)$ & 0.967 \\
\hline $\begin{array}{l}\text { Symptomatic patients } \\
\text { Model } 1^{\#}\end{array}$ & & & & \\
\hline $\begin{array}{l}\text { Non-Ob CT-LeSc > } 5 \\
\text { Model } 2^{\#}\end{array}$ & $2.63(1.07-6.46)$ & 0.035 & $2.39(1.29-4.42)$ & 0.006 \\
\hline $\begin{array}{r}\text { SIS }>5 \\
\text { Model } 3^{\S}\end{array}$ & - & - & $2.03(0.85-4.82)$ & 0.110 \\
\hline $\begin{array}{l}\text { Non-Ob CT-LeSc > } 5 \\
\text { Model } 4^{\S}\end{array}$ & $2.76(1.06-7.25)$ & 0.040 & $1.81(0.94-3.51)$ & 0.075 \\
\hline SIS $>5$ & - & - & $1.54(0.64-1.86)$ & 0.807 \\
\hline
\end{tabular}

$\S$ Adjusted for ASA and baseline clinical characteristics.

\# Adjusted for ASA and Morise score.

* Adjusted for ASA and Framingham score.

exhibited a prognosis that was halfway between that of patients without plaques and that of patients with a CT-LeSc $>5$.

\subsection{Future strategies for early detection of non-obstructive $C A D$}

Previous studies have proposed that early identification of nonobstructive CAD with CCTA may lead to a more aggressive strategy to control cardiovascular risk factors and to improve clinical follow-up [4, 20]. Recently, a sub-study of CONFIRM performed in a cohort of patients receiving baseline statin and aspirin treatment showed that statin therapy was associated with a significant mortality reduction in patients with non-obstructive CAD but had no impact on patients without CAD [18]. These are valid arguments in support of the identification of parameters, such as the CT-LeSc allowing to stratify the long-term prognosis of the large and heterogeneous group of patients in whom CCTA shows non-obstructive CAD. Moreover, recent studies demonstrated the prognostic value of plaque characterization by CCTA. Indeed, this non-invasive imaging modality is able to identify some features, such as vessel positive remodeling and low-attenuation plaques, that have been associated with a higher risk of cardiac events [19]. High-risk plaques can be detected by CCTA and are independent predictors of fatal and non-fatal acute coronary syndrome, while positive remodeling has been observed in coronary stenoses that, regardless of the degree of narrowing, were found to be functionally important by invasive fractional flow reserve [21]. Therefore, additional studies are needed to integrate the CT-LeSc with other features such as positive remodeling or eventually with non-invasive FFR by CCTA for improving the prognostic characterization of patients without obstructive coronary lesions.

\subsection{Study limitations}

In interpreting these data some limitations should be considered. First, management decisions in all patient, such as medical therapies or revascularization, were left to the discretion of the referring physicians. Because some therapies (i.e. aspirin, statin) may have a positive effect on patient outcomes and were commonly used in patients with and without plaques, we expect that differences between subgroups would be even greater in the absence of such treatments. Second, we included all-cause mortality in the primary and secondary endpoints given its unparalleled clinical importance and freedom from ascertainment bias. However, as specific causes of death for each patient were not uniformly available at all sites, the true proportion of deaths that could attributable to cardiovascular events in our patients is unknown. Third, this study included a cohort of patients who were referred for CCTA because of suspected CAD and were often symptomatic. Although it is unlikely that our patient symptoms were related to non-obstructive $\mathrm{CAD}$, generalization of this study findings to asymptomatic patients remains uncertain. Fourth, data on coronary calcium score were not included in the analysis because they were available for two third only of the study population.

\section{Conclusion}

The CT-adapted Leaman score is an independent predictor of major acute cardiac events and allows to distinguish, among a population with non-obstructive CAD, patients with risk of cardiac events similar to those without plaques from patients with a less favorable prognosis.

\section{Conflict of interest}

The authors report no relationships that could be construed as a conflict of interest.

\section{References}

[1] F. Bamberg, W.H. Sommer, V. Hoffmann, et al., Meta-analysis and systematic review of the long-term predictive value of assessment of coronary atherosclerosis by contrast-enhanced coronary computed tomography angiography, J. Am. Coll. Cardiol. 57 (24) (2011) 2426-2436.

[2] A. Pen, Y. Yam, L. Chen, et al., Discordance between Framingham risk score and atherosclerotic plaque burden, Eur. Heart J. 34 (14) (2013) 1075-1082.

[3] G.P. de Araújo, H.M. Garcia-Garcia, M.S. Carvalho, et al., Diabetes as an independent predictor of high atherosclerotic burden assessed by coronary computed tomography angiography: the coronary artery disease equivalent revisited, Int. J. Cardiovasc. Imaging 29 (5) (2013) 1105-1114.

[4] D. Andreini, G. Pontone, S. Mushtaq, et al., A long-term prognostic value of coronary CT angiography in suspected coronary artery disease, JACC Cardiovasc. Imaging 5 (7) (2012) 690-701.

[5] J.K. Min, A. Dunning, F.Y. Lin, et al., Age- and sex-related differences in all-cause mortality risk based on coronary computed tomography angiography findings results from the International Multicenter CONFIRM, J. Am. Coll. Cardiol. 58 (8) (2011) 849-860.

[6] S. Mushtaq, G.P. De Araujo, H.M. Garcia-Garcia, et al., Long-term prognostic effect of coronary atherosclerotic burden: validation of the computed tomography-Leaman score, Circ. Cardiovasc. Imaging 8 (2) (2015) e002332.

[7] J.K. Min, A. Dunning, F.Y. Lin, et al., Rationale and design of the CONFIRM (COronary CT Angiography EvaluatioN For Clinical Outcomes: An InteRnational Multicenter) Registry, J. Cardiovasc. Comput. Tomogr. 5 (2) (2011) 84-92.

[8] J. Leipsic, S. Abbara, S. Achenbach, et al., SCCT guidelines for the interpretation and reporting of coronary CT angiography: a report of the Society of Cardiovascular Computed Tomography Guidelines Committee, J. Cardiovasc. Comput. Tomogr. 8 (5) (2014) 342-358.

[9] S. Abbara, A. Arbab-Zadeh, T.Q. Callister, et al., SCCT guidelines for performance of coronary computed tomographic angiography: a report of the Society of Cardiovascular Computed Tomography Guidelines Committee, J. Cardiovasc. Comput. Tomogr. 3 (2) (2009) 190-204.

[10] W.G. Austen, J.E. Edwards, R.L. Frye, et al., A reporting system on patients evaluated for coronary artery disease. Report of the Ad Hoc Committee for Grading of Coronary Artery Disease, Council on Cardiovascular Surgery, American Heart Association, Circulation 51 (4Suppl) (1975) 5-40.

[11] G.P. de Araújo, H.M. Garcia-Garcia, H. Dores, et al., Coronary computed tomography angiography-adapted Leaman score as a tool to noninvasively quantify total coronary atherosclerotic burden, Int. J. Cardiovasc. Imaging 29 (7) (2013) 1575-1584.

[12] K. Thygesen, J.S. Alpert, H.D. White, et al., Joint ESC/ACCF/AHA/WHF Task Force for the Redefinition of Myocardial. Universal definition of myocardial infarction, J. Am. Coll. Cardiol. 50 (22) (2007) 2173-2195.

[13] H.S. Hecht, Coronary artery calcium scanning: past, present, and future, JACC Cardiovasc. Imaging 5 (8) (2015) 579-596.

[14] M.J. Pencina, R.B. D'Agostino Sr., E.W. Steyerberg, Extensions of net reclassification improvement calculations to measure usefulness of new biomarkers, Stat. Med. 30 (1) (2011) 11-21.

[15] J.E. van Velzen, J.D. Schuijf, F.R. de Graaf, et al., Diagnostic performance of noninvasive multidetector computed tomography coronary angiography to detect coronary artery disease using different endpoints: detection of significant stenosis vs. detection of atherosclerosis, Eur. Heart J. 32 (5) (2011) 637-645.

[16] M.S. Bittencourt, E. Hulten, B. Ghosh hajra, et al., Prognostic value of nonobstructive and obstructive coronary artery disease detected by coronary computed tomography angiography to identify cardiovascular events, Circ. Cardiovasc. Imaging 7 (2) (2014) 282-291.

[17] F.Y. Lin, L.J. Shaw, A.M. Dunning, et al., Mortality risk in symptomatic patients with nonobstructive coronary artery disease: a prospective 2-center study of 2,583 
patients undergoing 64-detector row coronary computed tomographic angiography, J. Am. Coll. Cardiol. 58 (5) (2011) 510-519.

[18] B.J. Chow, G. Small, Y. Yam, et al., Prognostic and therapeutic implications of statin and aspirin therapy in individuals with nonobstructive coronary artery disease: results from the CONFIRM registry, Arterioscler. Thromb. Vasc. Biol. 35 (4) (2015) 981-989.

[19] S. Motoyama, H. Ito, M. Sarai, et al., Plaque characterization by coronary computed tomography angiography and the likelihood of acute coronary events in mid-term follow-up, J. Am. Coll. Cardiol. 66 (4) (2015) 337-346.
[20] D. Andreini, G. Pontone, S. Mushtaq, et al., Prognostic value of multidetector computed tomography coronary angiography in diabetes: excellent long-term prognosis in patients with normal coronary arteries, Diabetes Care 36 (7) (2013) 1834-1841.

[21] Park HB, Heo R, ó Hartaigh B et al. Atherosclerotic plaque characteristics by CT angiography identify coronary lesions that cause ischemia: a direct comparison to fractional flow reserve. JACC Cardiovasc. Imaging 2015;8(1):1-10. 